\title{
VERIFICAÇÃO DAS PREVISÕES DE TEMPO PARA PRECIPITAÇÃO USANDO ENSEMBLE REGIONAL PARA O ESTADO DO CEARÁ EM 2009
}

\author{
CLEITON DA SILVA SILVEIRA ${ }^{1}$, ALEXANDRE ARAUJO COSTA ${ }^{1}$, MARIANE MENDES COUTINHO ${ }^{2}$, \\ FRANCISCO DE ASSIS DE SOUZA FILHO ${ }^{1}$, FRANCISCO DAS CHAGAS VASCONCELOS JÚNIOR ${ }^{3}$, \\ AURÉLIO WILDSON NORONHA ${ }^{3}$,
}

\author{
${ }^{1}$ Universidade Federal do Ceará, Fortaleza, CE, Brasil \\ ${ }^{2}$ Instituto Nacional de Pesquisas Espaciais, Centro de Ciências do Sistema Terrestre (INPE/CCST), São José dos Campos, SP, \\ ${ }^{3}$ Fundação Cearense de Meteorologia e Recursos Hídricos (FUNCEME), Fortaleza, CE, Brasil
}

cleitonsilveira16@yahoo.com.br, alexandrearaujoc@gmail.com,mariane_coutinho@yahoo.co.uk, assis@ufc.br, juniorphy@gmail.com, awildson@gmail.com

Recebido Junho de 2010 - Aceito Maio de 2011

\begin{abstract}
RESUMO
Este artigo apresenta um estudo de verificação das previsões de chuva de um sistema de previsão do tempo por ensemble regional. O conjunto é composto por seis membros, dos quais quatro utilizam o modelo RAMS 6.0 e dois usam o WRF 3.1, inicializados com dados dos modelos globais do CPTEC ou GFS e diferentes parametrizações de convecção. A verificação foca nas previsões de chuva de 24, 48 e 72 horas e nos limiares de precipitação de $1 \mathrm{~mm}, 5 \mathrm{~mm}$ e $10 \mathrm{~mm}$ sobre o Estado do Ceará. Os membros do ensemble apresentaram resultados superiores à persistência em todo o domínio avaliado. O modelo RAMS apresenta maior índice de acerto, principalmente no litoral norte do Estado, porém um maior falso alarme em comparação com o modelo WRF. O sistema de previsão de chuva diminui sua qualidade com o aumento dos horizontes e a intensidade da chuva que se quer prever.
\end{abstract}

Palavras-chave: Previsão por ensemble, modelos numéricos de tempo, verificação.

\begin{abstract}
VERIFICATION OF RAINFALL WEATHER FORECASTS USING REGIONAL ENSEMBLE FOR CEARÁ STATE IN 2009

This work presents a verification study of rainfall forecast by a regional model ensemble prediction system. The ensemble is formed by six members; four of them using RAMS 6.0, and two using WRF 3.1, all initialized by CPTEC or GFS general circulation model data and making use of different convection parameterizations. The verification focuses on rainfall forecasts for 24,48 and 72 hours and rainfall thresholds of $1 \mathrm{~mm}, 5 \mathrm{~mm}$ and $10 \mathrm{~mm}$ over the Ceará State. All ensemble members have better skill than persistence in the entire verification domain. RAMS shows a larger accuracy, especially at the northern coast of Ceará, but gives also a larger false alarm ratio than WRF model. The quality of the rainfall forecasts system decreases as either, the rainfall intensity or the forecast horizon time increase.
\end{abstract}

Keywords: Ensemble forecasts; Numerical models; Verification.

\section{INTRODUÇÃO}

As previsões numéricas de precipitação fornecem informações importantes para diversos setores da sociedade, principalmente para aqueles sensíveis a essa variável atmosférica, como a agricultura, recursos hídricos, etc. Além disso, a possibilidade de prever eventos extremos permite que decisões possam ser tomadas para que os impactos de tais eventos sejam minimizados, reduzindo assim o grau de vulnerabilidade de uma determinada região.
As previsões individuais realizadas com modelos atmosféricos são utilizadas por vários centros de meteorologia (Bustamante et. al., 2005 e Moura et. al, 2010), porém as previsões determinísticas, podem apresentar resultados que podem diferir bastante da realidade e entre si. Isto se deve ao comportamento caótico da atmosfera (Lorenz, 1965) e às aproximações físicas associadas ao modelo (métodos numéricos ou parametrizações físicas). No caso de modelos regionais, também existe incerteza proveniente das condições fornecidas pelo modelo global para as fronteiras laterais do modelo (Warner 
et al. ,1997 e Hou et al, 2001). As incertezas associadas às condições iniciais da atmosfera diminuem a confiabilidade da previsão determinística, mostrando a necessidade de métodos probabilísticos para descrever adequadamente o comportamento caótico da atmosfera (Coutinho, 1999).

A técnica de previsão por conjunto (ensemble) surgiu como uma forma de tratar erros cometidos na geração das condições iniciais da atmosfera e causados por deficiências dos modelos, buscando ampliar o prazo de previsão do modelo e obter melhores previsões numéricas de tempo. Essa técnica considera uma série de condições iniciais distintas (Leith, 1974, Machado et al.,2010), ou um conjunto de situações com o modelo ligeiramente modificado (Stensurd et al, 1998), ou ainda, previsões a partir de um conjunto de modelos distintos (Krishnamurti e Zhang., 1999; Hou et al., 2001;Silveira et al.,2010b; Silveira et al.,2010c).

A destreza de um sistema de previsão depende da incerteza associada ao sistema meteorológico que se pretende prever e das características do próprio modelo ou conjunto de modelos (resolução espacial, qualidade da representação dos processos físicos, número de membros, qualidade das condições iniciais assimiladas etc). Melhorar o entendimento em relação à confiabilidade, à sensibilidade e às limitações do sistema de previsão é crucial para que o mesmo possa se constituir enquanto ferramenta para definir políticas de planejamento e gestão (Reis Júnior et al., 2009).

O objetivo deste trabalho é apresentar um sistema de previsão do tempo para o estado do Ceará e identificar as regiões de maior e menor previsibilidade, usando uma técnica de ensemble regional. Os modelos numéricos empregados neste conjunto foram avaliados para as previsões dede $24 \mathrm{~h}, 48 \mathrm{~h} \mathrm{e}$ $72 \mathrm{~h}$, com base nas medições pluviométricas disponibilizadas pela Fundação Cearense de Meteorologia e Recursos Hídricos (FUNCEME).

\section{MATERIAIS E MÉTODOS}

\subsection{0 ensemble regional}

$\mathrm{Na}$ implementação da técnica de previsão de tempo por conjunto para o estado do Ceará são utilizados os modelos regionais RAMS 6.0 (Regional Atmospheric Modeling System) (Pielke et al., 1992; Cotton et al., 2003) e WRF 3.1 (Weather Research and Forecasting) (Skamarock et al., 2005), inicializados com dados dos modelos globais do Centro de Previsão de Tempo e Estudos Climáticos (CPTEC) (T126L28) ou Global Forecast System (GFS) com resolução espacial de $1^{\circ}$ por $1^{\circ}$. O método empregado para geração do conjunto de previsões consiste na utilização de combinações de diferentes configurações físicas e distintas condições iniciais e de fronteira para cada rodada dos modelos.

Todas as simulações realizadas utilizam uma grade horizontal de 115 por 115 pontos com aproximadamente 25 $\mathrm{km}$ de resolução, centrada no estado do Ceará $\left(39,0^{\circ} \mathrm{W} ; 6,5^{\circ} \mathrm{S}\right)$ cobrindo boa parte do Nordeste brasileiro, assim como uma porção do Oceano Atlântico.

$\mathrm{O}$ ensemble regional de previsão numérica de tempo operacional na FUNCEME consiste em quatro combinações de configuração do modelo RAMS, aliadas a duas combinações de configurações do modelo WRF, totalizando assim seis membros, conforme a Tabela 1 .

Os membros que utilizam o modelo regional RAMS foram obtidos com a combinação de duas opções de parametrização de convecção e de duas fontes distintas de forçantes de grande escala. Já os que utilizam o modelo regional WRF foram obtidos com duas opções de parametrização de convecção a partir do mesmo forçante de grande escala.

Os esquemas de convecção usados no modelo RAMS são os propostos por Kuo (1974) modificado por Tremback (1990), baseado na convergência do vapor e ajuste convectivo, e outro, por Kain e Fritsch (1993), baseado no fluxo de massa na base das nuvens. Com relação ao modelo WRF, as parametrizações de convecção usadas são as propostas por Grell e Devenyi (2002) e Kain (2004). Em ambos os modelos regionais os esquemas de convecção são acionados a cada 20 minutos do período simulado.

Para os membros que utilizam o modelo regional WRF, foram utilizadas como condições de contorno os dados provenientes do modelo global do GFS do National Centers

Tabela 1 - Membros do ensemble

\begin{tabular}{cccc} 
Membros do ensemble & Modelo regional & Modelo global & Parametrização de convecção \\
\hline RGKF & RAMS 6.0 & GFS & Kain-Fritsch \\
RGKU & RAMS 6.0 & GFS & Kuo \\
RCKF & RAMS 6.0 & CPTEC & Kain-Fritsch \\
RCKU & RAMS 6.0 & CPTEC & Kuo \\
WGKF & WRF 3.1 & GFS & Kain-Fritsch \\
WGGR & WRF 3.1 & GFS & Grell \\
\hline
\end{tabular}


for Environmental Prediction (NCEP), com as análises das 0000 UTC e as previsões de 3 em 3 horas para a definição das condições de fronteira. Os dados têm uma resolução em latitude e longitude de $1^{\circ}$, além dos dados de superfície e de 4 camadas no subsolo.

Já para os membros em que se usou o modelo regional RAMS foram utilizados os modelos globais do GFS ou CPTEC. Os modelos regionais diferem-se na assimilação de dados de superfície. O RAMS e o WRF utilizam valores estáticos da topografia, albedo, categoria do solo, cobertura vegetal, dentre outros, retirados das bases de dados do USGS (United States Geological Survey), porém o RAMS assimila também a temperatura da superfície do mar (TSM) de estimativas de satélites fornecidas por Remote Sensing Systems (RSS).

As condições iniciais e de contorno, utilizadas nos membros que assimilam dados provenientes do modelo global do CPTEC, possuem resolução horizontal de aproximadamente $100 \times 100 \mathrm{~km}$ próximo ao equador e 28 níveis verticais (T126L28), sendo assimiladas a cada 6 horas.

A forma mais simplificada de obter informações da previsão por conjunto é o ensemble médio, que consiste em calcular a média das previsões, considerando que todos os membros possuam a mesma probabilidade de representarem o estado futuro da atmosfera.

O ensemble médio para cada ponto de grade $\mathrm{j}$ é dado por

$$
\mathrm{EMj}=\frac{1}{N} \sum_{i=1}^{N} F_{j}^{i}
$$

onde $N$ é o número de membros do ensemble e $F_{j}^{i}$ são as previsões de cada membro.

$\mathrm{O}$ ensemble médio, em geral, fornece uma previsão melhor do que cada elemento do conjunto (Toth e Kalnay, 1993), já que alguns erros nas previsões individuais devem se cancelar na média, desde que o conjunto represente adequadamente a incerteza associada à condição inicial.

\subsection{O modelo de persistência}

O modelo de persistência consiste em obter previsões a partir de valores observados num dado período anterior à data de validade da previsão. Esse modelo admite que a observação num dado período seja reproduzida novamente no futuro (Carvalho et. al, 2008 e Reis Júnior, 2009). Por exemplo, o modelo de persistência de $24 \mathrm{~h}$ será o valor observado $24 \mathrm{~h}$ antes do período de interesse.

\subsection{Verificações das previsões}

As simulações realizadas com os modelos compreendem o período de 01 de fevereiro de 2009 a 31 de maio de 2009 , correspondendo a 120 pares modelado-observado para cada membro. Para avaliar a destreza dos modelos, comparam-se os valores simulados de precipitação acumulada diária de 7 horas a 7 horas do dia seguinte, com os dados de 480 pluviômetros espalhados por todo o estado do Ceará, conforme mostra a Figura 1, interpolados para a grade dos modelos regionais.

A precipitação interpolada para os pontos da grade constitui-se de uma media ponderada das precipitações observadas (Equação 2), tal que as funções-peso são dadas conforme a Equação 3.

$$
\begin{aligned}
& P_{\text {grade }}=\frac{\sum_{N=1}^{N=480} W_{N} P_{N}}{\sum_{N=1}^{N=480} W_{N}} \\
& W_{N}=\exp \left(-\sqrt{\left(\frac{X_{N}-X_{\text {grade }}}{K_{X}}\right)^{2}+\left(\frac{X_{N}-X_{\text {grade }}}{K_{Y}}\right)^{2}},\right)
\end{aligned}
$$

se $W_{N}>\exp \left(-R^{2}\right)$, senão $W_{N}=0$; onde $X_{\text {grade }}$ e $Y_{\text {grade }}$ são as coordenadas dos pontos de grade modelo; $X_{N}$ e $Y_{N}$ são as coordenadas dos pluviômetros; $N$ é o número referente ao pluviômetro; $P_{N}$ são as precipitações observadas em cada pluviômetro; $W_{N}$ são os pesos de cada pluviômetro em relação a um dado ponto de grade; $P_{\text {grade }}$ são as precipitações de cada ponto de grade depois da interpolação; $K_{X}, K_{Y} \mathrm{e} R$ são constantes.

Para as constantes $K_{X}, K_{Y}$ e $R$ foi atribuído o valor de 0,45 . Esse valor foi selecionado depois de alguns testes, de modo que a precipitação observada pudesse representar praticamente todos os pontos de grade modelados pertencentes ao estado do Ceará.

Na Tabela 2 é apresentada a contingência $2 \times 2$, a qual expressa o número de vezes em que ocorreram as diversas combinações entre previsão e observação. No caso específico da tabela $2 \times 2$, os valores possíveis de precipitação são divididos em duas categorias: abaixo de um limite estabelecido e acima desse limite. Os valores de a, b, c e d são utilizados para estimar as frequências relativas de cada uma destas combinações. As métricas utilizadas neste trabalho são simplificadamente descritas a seguir (maiores detalhes podem ser encontrados em Wilks, 1995).

A proporção de acerto $(\mathrm{H})$ expressa a fração na qual o sistema de previsão acertou a faixa ou categoria de chuva. H é calculado da seguinte forma:

$$
H=\left(\frac{a+d}{n}\right) \times 100
$$

onde $\mathrm{n}$ é o número de dias do período de verificação. Se o sistema de previsão for perfeito, $\mathrm{H}=100 \%$. A proporção de 


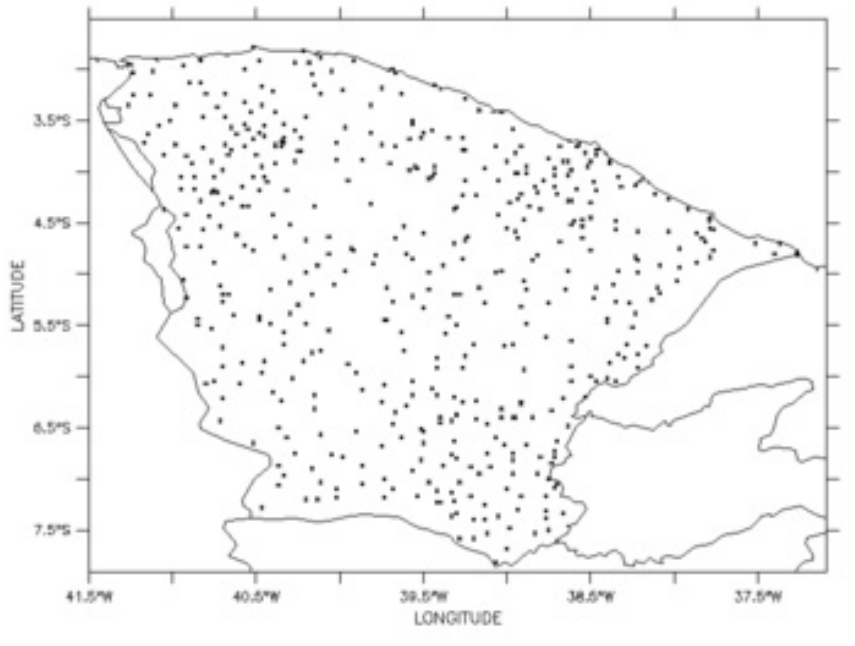

Figura 1 - Localização dos pluviômetros usados no estudo.

Tabela 2 - Tabela de contingência 2 × 2

\begin{tabular}{|c|c|c|c|}
\hline & & \multicolumn{2}{|c|}{ Observação } \\
\hline \multirow{3}{*}{ Previsão } & & Sim & Não \\
\hline & Sim & $\mathrm{a}$ & $b$ \\
\hline & Não & $\mathrm{c}$ & $d$ \\
\hline
\end{tabular}

acertos não faz qualquer distinção entre acertar a precipitação acima de um limite pré-estabelecido e acertar a não ocorrência do evento. Segundo Murphy (1996) esta métrica não é interessante, quando a probabilidade de ocorrência do evento que se deseja prever, for pequena. Para este caso, um modelo que prevê sempre a não ocorrência do evento pode resultar num $\mathrm{H}$ bastante elevado. Porém, em casos como este, obter um H maior não caracteriza muito bem o sistema de previsão, já que não há utilidade em prever sempre a não ocorrência de um evento.

A probabilidade de detecção $(P O D)$ representa a probabilidade do sistema prever a ocorrência do evento, dado que o evento de fato ocorreu. Um sistema de previsão perfeito possui $\mathrm{POD}=100 \%$, ou seja, o evento nunca acontece sem que o sistema de previsão o tenha previsto.

$$
P O D=\left(\frac{a}{a+c}\right) \times 100
$$

O falso alarme (FAR) representa a probabilidade de não se observar chuva acima de um determinado limite, dado que o sistema de previsão indicou chuva acima dessa categoria. Um sistema de previsão perfeito apresenta $\mathrm{FAR}=0$, da mesma forma que se o sistema sempre indica que o evento vai ocorrer e ele não ocorre, tem-se $\mathrm{FAR}=100 \%$. O FAR é dado por:

$$
F A R=\left(\frac{b}{a+b}\right) \times 100
$$

A razão de VIÉS (BIAS em inglês) indica uma possível tendência do sistema de previsão em superestimar ou subestimar os eventos de precipitação, sendo calculada como a razão entre o total de eventos previstos e o total de eventos observados, ou seja,

$$
V I E ́ S=\frac{a+b}{a+c},
$$

de modo que VIÉS = 1 significa que o sistema de previsão não é tendencioso, ou seja, não possui tendência a subestimar ou superestimar o número de eventos de precipitação acima do limiar estabelecido. Para VIÉS maior que 1, o sistema de previsão tende a superestimar o número de eventos ocorrido para um dado limiar, assim como para VIÉS menor que 1 o sistema tende a subestimar.

\section{RESULTADOS E DISCUSSÃO}

Na Figura 2 é apresentado o índice de acerto $(\mathrm{H})$ dos membros do ensemble, ensemble médio e persistência para os limiares de $1 \mathrm{~mm}$ (a), $5 \mathrm{~mm}$ (b) e $10 \mathrm{~mm}$ (c), para a previsão de $24 \mathrm{~h}$. Todos os modelos apresentam melhores resultados do que a persistência, principalmente no litoral do estado do Ceará, onde o seu índice de acerto é superior a 75\% para o limiar de $1 \mathrm{~mm}$. À medida que o limiar de chuva adotado na construção da tabela de contingência aumenta, a qualidade das previsões diminui, mas continua melhor do que a persistência. Exceto para região mais ao sudoeste do Ceará para $10 \mathrm{~mm}$, onde a persistência apresentou índice de acerto superior aos demais modelos. Isto pode estar associado à baixa frequência de ocorrência desse evento nessa região.

Analisando a proporção de acerto, ainda na Figura 2a, observa-se que os membros que utilizam a parametrização de Kain-Fritsch (RCKF, RGKF e WGKF) apresentam melhores resultados na região mais ao sul do Ceará em detrimento às outras simulações (RCKU, RGKU e WGGR) para o limiar de $1 \mathrm{~mm}$.

Na Figura 3 são comparados dois membros que possuem modelos regionais distintos, WGKF e RGKF, observa-se que o RAMS apresentou uma maior proporção de acerto (conforme Figura 2) e uma maior probabilidade de detecção para praticamente todo o domínio avaliado para o limiar de $1 \mathrm{~mm}$, porém o modelo WRF apresentou um falso alarme menor. O modelo RAMS tende a superestimar a ocorrência de eventos de precipitação, principalmente na região sul do estado, apresentando VIÉS bastante elevado, enquanto esta métrica para 

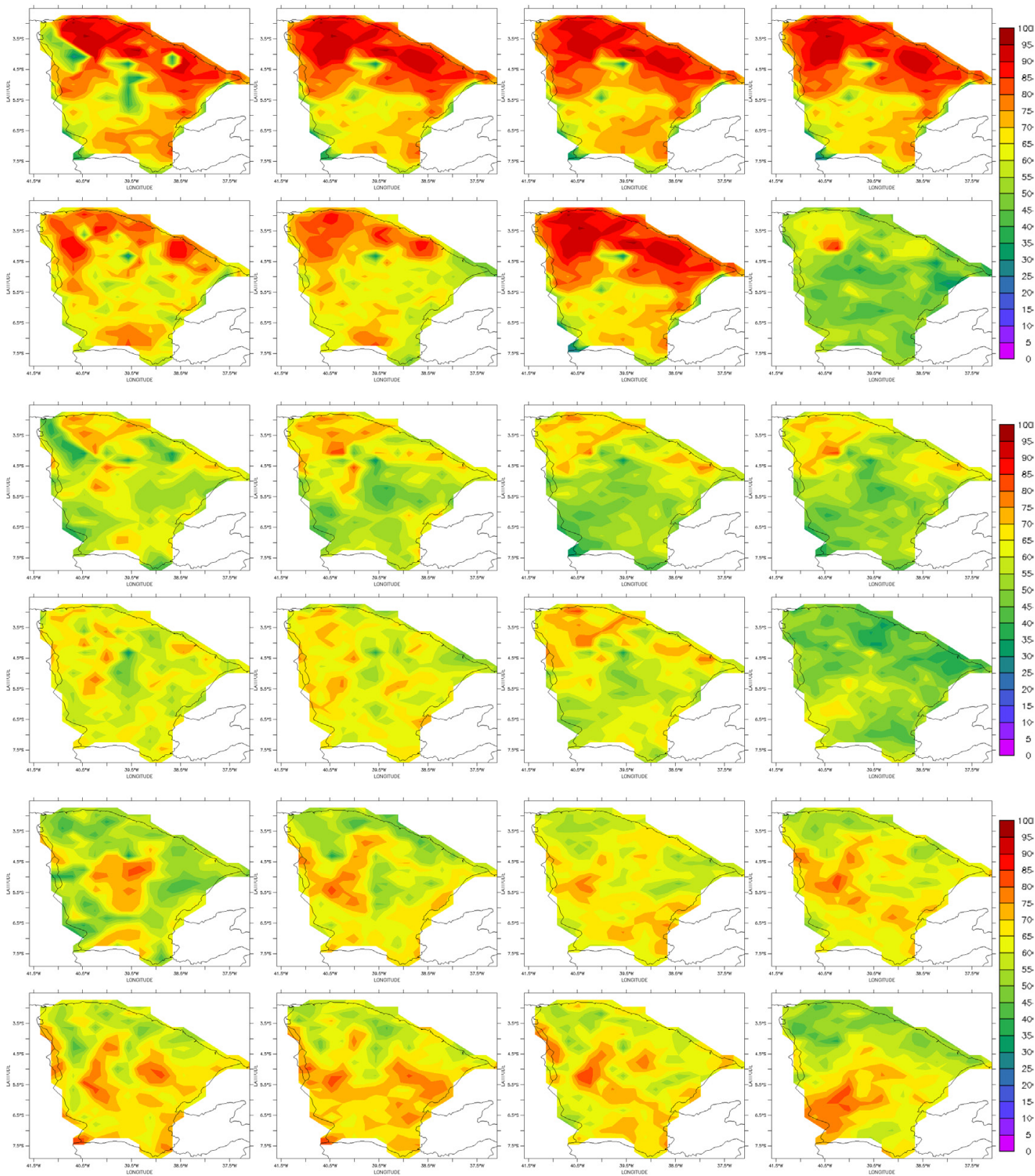

Figura 2 - Verificação das previsões de 24 horas para os limiares de: (a) $1 \mathrm{~mm}$; (b) $5 \mathrm{~mm}$ e (c)10 mm. O índice de acerto(H) é mostra para cada modelo na seguinte sequência (da esquerda para direita e de cima para baixo): RCKF, RCKU, RGKF, RGKU, WGKF, WGGR, EM e Persistência.

o WRF é muito próxima de 1 , em quase todo o domínio para os limiar de $1 \mathrm{~mm}$, esse comportamento também é seguido para o limiar de $5 \mathrm{~mm}$.

Na Figura 2 o membro utilizado no modelo RAMS e a parametrização de convecção de Kain-Fritsch, com forçante de grande escala do modelo global do CPTEC (RCKF), apresentaram piores resultados no sertão central e no litoral oeste do estado, quando comparado ao membro que assimilou dados do GFS (RGKF). Isto fica evidente quando compara-se, na Figura 4, o VIÉS desses dois membros para o limiar de 10 mm e para previsão de $24 \mathrm{~h}$. O RCKF superestimam a ocorrência de precipitação em algumas regiões, mas próximo a estas, apresentam VIÉS aproximadamente nulo, principalmente para o limiar de $10 \mathrm{~mm}$. Isto sugere a existência de um erro sistemático na localização dos eventos de precipitação.

Na Figura 5 são apresentados $H, F A R, P O D$ e VIÉS dos membros do ensemble, ensemble médio e persistência para os limiares de $5 \mathrm{~mm}$ (a) e $10 \mathrm{~mm}$ (b), para a previsão de 72h. Para as previsões $72 \mathrm{~h}$ os modelos apresentam melhores previsões do que a persistência, com uma proporção de acertos bem acentuada 

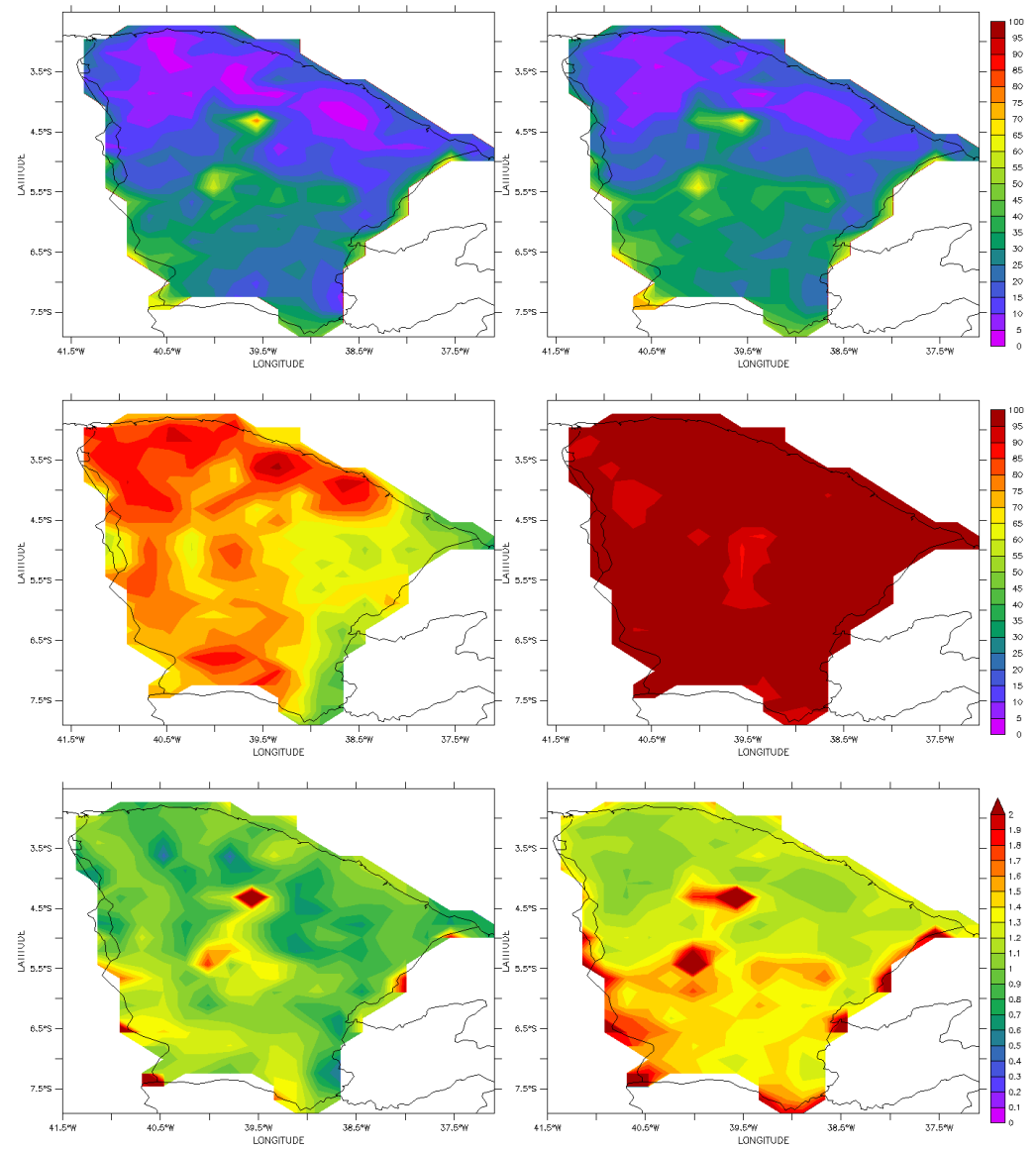

Figura 3 - Verificação das previsões de 24 horas para o limiar de 1 mm: (a)FAR; (b)POD e (c) VIÉS. Modelo a esquerda WGKF e a direita RGKF.
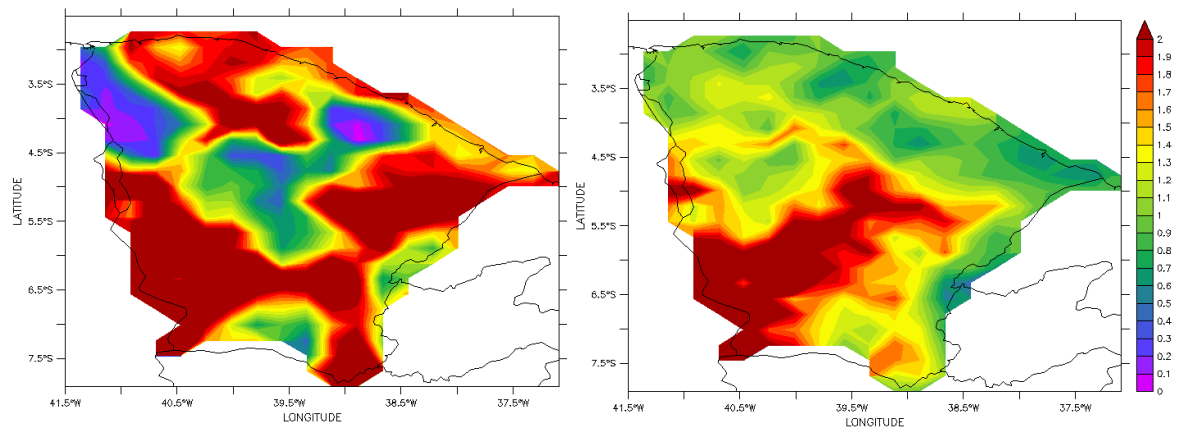

Figura 4 - BIAS das previsões de 24 horas para o limiar de $10 \mathrm{~mm}$. Modelo a esquerda RCKF e a direita RGKF.

no litoral norte do estado. Porém, há uma sensível redução da qualidade para previsões mais distantes.

Para o limiar de $10 \mathrm{~mm}$ das previsões de $72 \mathrm{~h}$, apesar de não apresentar índice de acerto melhor que o WGGR, WGKF e a persistência, o ensemble médio possui FAR menor que os membros que usam o RAMS e um POD superior ao WRF. O que indica que esta média das previsões reduz o comportamento de superestimação de eventos de chuva desse modelo e mantém ainda uma informação de qualidade, evidenciada pelos valores elevados de POD. Isto sugere que a média é uma ferramenta potencial para limiares mais elevados e para previsões mais afastadas das condições iniciais (Silveira, 2009).

A persistência possui extrema dificuldade de detectar chuvas para o limiar de $10 \mathrm{~mm}$, apresentando POD inferior a $20 \%$ e um VIÉS muito próximo de zero em praticamente todo o domínio para os três limites de previsões. 


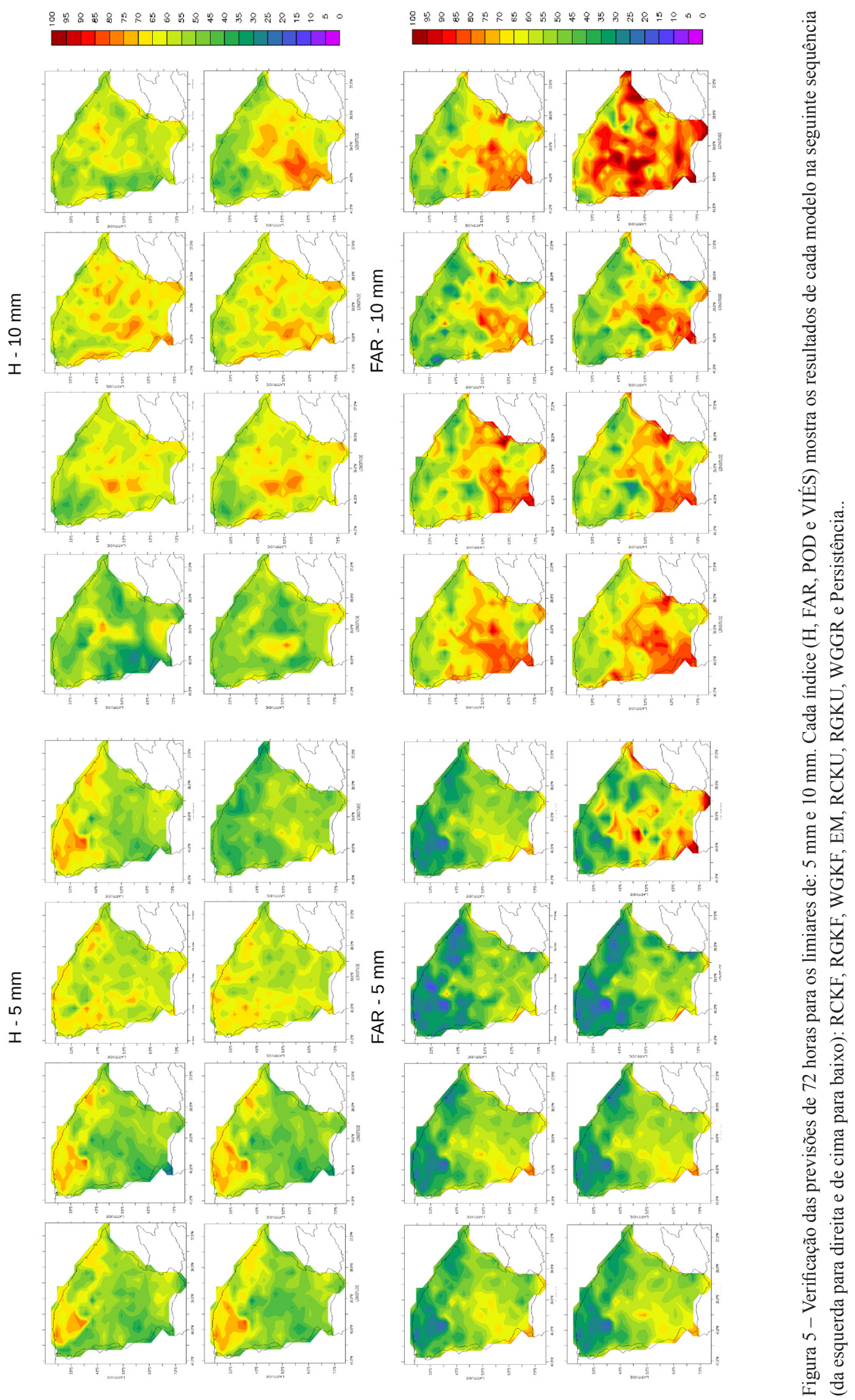



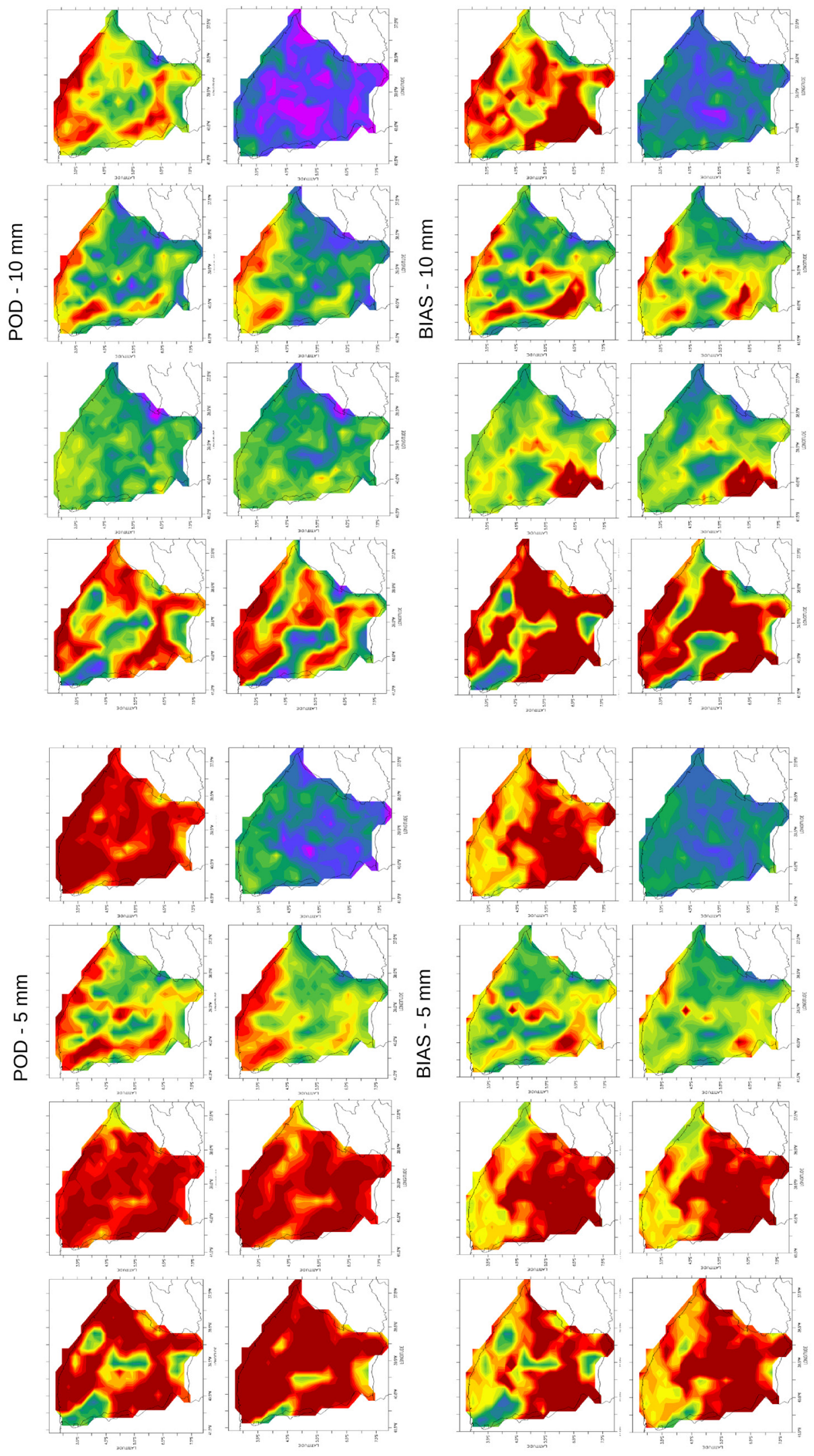

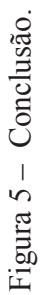




\section{CONCLUSÕES}

Todos os membros do conjunto apresentaram resultados melhores que a persistência, principalmente no litoral do estado. À medida que o limiar de chuva adotado na construção da tabela de contingência aumenta, a qualidade das previsões diminui, mas continua melhor que a persistência.

Em geral, o modelo RAMS demonstrou maior probabilidade de detecção e um VIÉS elevado para 1 e $5 \mathrm{~mm}$ de limiar, principalmente na região mais ao sul do estado. Isso indica que esse modelo tende a superestimar a ocorrência de eventos de precipitação. O modelo WRF, apesar de apresentar um índice de acerto e uma probabilidade de detecção menor que o RAMS, apresenta um menor falso alarme, o que pode justificar a sua utilização operacional.

Os membros que utilizam o modelo global do CPTEC como forçante de grande escala apresentam VIÉS bastante elevado em algumas regiões, indicando uma tendência do modelo superestimar os eventos de chuva observados, principalmente para o limiar de $10 \mathrm{~mm}$.

O modelo de persistência possui dificuldade de detectar chuva, principalmente para os limiares de $5 \mathrm{~mm}$ e $10 \mathrm{~mm}$, o que torna inviável o seu uso para fornecer informações para alerta de eventos extremos ou para modelos hidrológicos do tipo chuva-vazão.

O sistema de previsão numérica por ensemble mostra-se eficiente em detectar a ocorrência ou não de chuva diária, apresentando resultados mais satisfatórios na região litorânea do estado do Ceará, possivelmente associados a uma representação adequada da circulação sobre essa região pelo sistema de previsão e/ou uma boa representação do ciclo diurno de precipitação nessa região (Silveira et.al., 2010a).

\section{REFERÊNCIAS}

BUSTAMANTE, J. F.; CHOU, S. C.; ROZANTE, J. R.; GOMES, J. L.. Uma avaliação da previsibilidade de tempo do modelo ETA para a América do Sul. Revista Brasileira de Meteorologia, v. 20, n. 1, p. 59-70, 2005.

CARVALHO, T. L. L.; REIS, D. S. JR.; MARIA, P. H. S.; SOMBRA, S. S; COSTA, A. A.; SANTOS, A. C. S.. "Avaliação da Previsão de Curto Prazo de Chuva na Bacia do Alto Jaguaribe durante a Cheia de 2008", In: Anais do IX Simpósio de Recursos Hídricos do Nordeste, 2008, Salvador - BA. Anais do XVIII Simpósio Brasileiro de Recursos Hídricos, 2008.

COTTON, W. R.; PIELKE SR, R. A.; WALKO, R. L.; ET AL. RAMS 2001: Current status and future directions. Meteorology and Atmospheric Physics, v. 82, n. 1, p. 5-29, 2003.

COUTINHO, M.M. Previsão por conjuntos utilizando perturbações baseadas em componentes principais. 1999. 136f. Dissertação
(Mestrado em Meteorologia) - Instituto Nacional de Pesquisas Espaciais INPE, São José dos Campos, SP, 1999.

GRELL, G. A.; DEVENYI, D. A generalized approach to parameterizing convection, combining ensemble and data assimilation techniques. Geophysical Research Letters,v. 29, n. 14, p. 1693, 2002.

HOU, D. et al. Objective verification of the samex'98 Ensemble forecast. Monthly Weather Review, v. 129, p. 73-91, 2001.

KAIN, J. S.; FRITSCH, J. M. Convective parameterization for mesoscale models: The Kain-Fritsch scheme. The Representation of Cumulus Convection in Numerical Models. Meteorological Monographs, v. 24, p. 165-170, 1993.

KAIN, S. J. The Kain-Fritsch Convective Parameterization: An Update. Journal of Applied Meteorology, v. 43, p. 170-181, 2004.

KRISHNAMURTI; T.N. ZHANG, Z.. A perturbation method for hurricane ensemble predictions. Monthly Weather Review, v. 127, n. 4, p. 447-469, 1999.

KUO, H. L. Further Studies of the Parameterization of the Influence of Cumulus Convection on Large-Scale Flow. Journal of the Atmospheric Sciences, v. 31, n. 5, p. 1232-1240, 1974.

LEITH,C.E Theoretical Skill of Monte Carlo Forescast. Monthly Weather Review, v. 102, n. 6, p. 409-418,1974.

LORENZ, E. N A study of the predictability of a 28 -Variable Atmospheric Model. Tellus, v. 17, n. 3, p. 321-333, 1965.

MACHADO, L. H. R.; MENDONÇAA. M.; MENDONÇA, R. W. B.; BONATTI, J. P.. Impacto da utilização de previsões "defasadas" no sistema de previsão de tempo por conjunto do CPTEC/INPE. Revista Brasileira de Meteorologia, v. 25, n. 1, p. 54-69, 2010. MOURA, R. G.; HERDIES, D. L.; MENDES, D.; MENDES, M. C. D.. Avaliação do modelo regional ETA utilizando as análises do CPTEC e NCEP. Revista Brasileira de Meteorologia, v. 25, n. 1, 46-53, 2010.

MURPHY, A. H.. "The Finley affair: A signal event in the history of forecast verification", Weather and Forecasting, v. 11, n. 1, p. 3-20, 1996

PIELKE, R. A.; COTTON, W. R.; WALKO, R. L.; ET AL. A comprehensive meteorological modeling system-RAMS. Meteorology and Atmospheric Physics, v. 49, n. 1, p. 69-91, 1992. REIS JUNIOR, D. S.; SOMBRA, S. S.; COSTA, A. A.; NETO, O.L.;SANTOS, A.C.S.; FIRMINO, M.B.M.. Verificação da Previsão Operacional de Chuva sobre Bacias Hidrográficas do Estado do Ceará. In: XVIII Simpósio Brasileiro de Recursos Hídricos, 2009, Campo Grande. Anais do XVIII Simpósio Brasileiro de Recursos Hídricos, 2009.

SILVEIRA, C. S. Previsão de Tempo por Ensemble Regional para o Nordeste Brasileiro. Dissertação (Mestrado Acadêmico em Ciências Físicas Aplicadas) - Universidade Estadual do Ceará, Fortaleza, 2009.

SILVEIRA, C.S. ; COSTA, A. A. ; VASCONCELOS JUNIOR, F. C. ; MENEZES NETO, O. L. . Can RCMs Properly Represent the Diurnal Cycle of Precipitation in the Tropics? Evaluating the 
Northeast Brazil Case. In: The Meeting of the Americas, 2010, Foz do Iguaçu. Atmospheric Sciences, 2010a.

SILVEIRA,C.S.; COUTINHO, M.M.; COSTA,A.A.; VASCONCELOS JUNIOR, F.C. . Previsão de tempo por conjuntos para a região Nordeste do Brasil: uma avaliação para precipitação;. In: XVI Congresso Brasileiro de Meteorologia, 2010, Belém. Modelagem Atmosférica, $2010 \mathrm{~b}$.

Silveira,C.S. ; REIS JUNIOR, D. S. ; FIRMINO, M. B. M. ; COSTA, A. A. . Verification of the Regional Ensemble Precipitation Forecast System for the State of Ceara, Brazil. In: The Meeting of the Americas, 2010, Foz do Iguaçu. Hydrology, 2010c.

SKAMAROCK, W.C. et al. A description of the Advanced Research WRF version 2. NCAR Tech Notes-468+STR, 2005.

STENSURD,D.J.,J.-W.BAO,AND T.1 WARNER. Ensemble Forcasting of mesoscale convective system. Preprints, 12th conf. numerical weather prediction, Phoenix, AZ, American Meteorological Society, p. 265-268, 1998.
TREMBACK, C. Numerical Simulation of a Mesoscale Convective Complex: Model Development and Numerical Results. 289f. 1990. Tese (Department of Atmospheric Science) - Colorado State University, 1990

TOTH, Z.; KALNAY, E. Ensemble Forecasting at NMC: The Generation of Perturbations. Bulletin of the American Meteorological Society, v. 74, n. 12, p. 2317-2339, Dec. 1993.

WARNER, T. T.; R. A. PETERSON; R. E. TREADON: A tutorial on lateral boundary conditions as a basic and potentially serious limitation to regional weather prediction. Bulletin American Meteorological Society, v. 78, p. 2599-2617,1997.

WILKS, D.S Statistical Methods in the Atmospheric Science. San Diego: Academic Press, 467p, 1995. 